\title{
Towards Automated, Provenance-Driven Security Audit for git-Based Repositories: Applied to Germany's Corona-Warn-App: Vision Paper
}

\author{
Tim Sonnekalb \\ Thomas S. Heinze \\ German Aerospace Center (DLR), \\ Institute of Data Science \\ Jena, Germany \\ tim.sonnekalb@dlr.de \\ thomas.heinze@dlr.de \\ Jesus M. Gonzalez-Barahona \\ Universidad Rey Juan Carlos \\ Fuenlabrada, Spain \\ jgb@gsyc.urjc.es
}

\author{
Lynn von Kurnatowski \\ German Aerospace Center (DLR), \\ Institute for Software Technology \\ Weßling, Germany \\ lynn.kurnatowski@dlr.de
}

\author{
Andreas Schreiber \\ German Aerospace Center (DLR), \\ Institute for Software Technology \\ Köln, Germany \\ andreas.schreiber@dlr.de
}

\begin{abstract}
Software repositories contain information about source code, software development processes, and team interactions. We combine provenance of the development process with code security analysis to automatically discover insights. This provides fast feedback on the software's design and security issues, which we evaluate on projects that are developed under time pressure, such as Germany's COVID-19 contact tracing app 'Corona-Warn-App'.
\end{abstract}

\section{CCS CONCEPTS}

- Security and privacy $\rightarrow$ Software security engineering; • Software and its engineering $\rightarrow$ Software libraries and repositories; Software defect analysis; • Information systems $\rightarrow$ Data mining; $\bullet$ Human-centered computing $\rightarrow$ Open source software.

\section{KEYWORDS}

program analysis, provenance, software security, repository mining, open source software, covid-19

\section{ACM Reference Format:}

Tim Sonnekalb, Thomas S. Heinze, Lynn von Kurnatowski, Andreas Schreiber, Jesus M. Gonzalez-Barahona, and Heather Packer. 2020. Towards Automated, Provenance-Driven Security Audit for git-Based Repositories: Applied to Germany's Corona-Warn-App: Vision Paper. In Proceedings of the 3rd ACM SIGSOFT International Workshop on Software Security from Design to Deployment (SEAD '20), November 9, 2020, Virtual, USA. ACM, New York, NY, USA, 4 pages. https://doi.org/10.1145/3416507.3423190

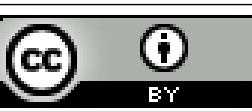

This work is licensed under a Creative Commons Attribution International 4.0 License SEAD '20, November 9, 2020, Virtual, USA

(c) 2020 Copyright held by the owner/author(s)

ACM ISBN 978-1-4503-8126-0/20/11.

https://doi.org/10.1145/3416507.3423190

\author{
Heather Packer \\ University of Southampton \\ Southampton, United Kingdom \\ hp3@ecs.soton.ac.uk
}

\section{INTRODUCTION}

Software repositories contain much information besides the source code itself. Especially for Open Source projects, the team composition and development process is transparent and traceable and can be evaluated at any point of time by, for example, continuous evaluation with regards to security by automated analysis [8].

The COVID-19 pandemic raises challenges for scientists of many disciplines. Computer scientists and software developers help to fight the pandemic with software systems, which must be developed under time pressure [2], with high quality, and with accepted concepts for data security and privacy.

For example, apps for mobile devices that support contact tracing of infected persons are useful to identify local COVID-19 hot-spots and find other persons, who are potentially infected, too. For contact tracing, several architectures are possible and have been discussedsometimes very controversial-in many countries. Two favoured approaches are centralized and decentralized architectures; both using Bluetooth Low Energy for contact identification. Apple and Google developed an Exposure Notification API ${ }^{1}$ as extension of their operating systems iOS and Android, which developers of exposure notification apps can use for privacy-preserving contact tracing. We focus on the German decentralized exposure notification app Corona-Warn-App ${ }^{2}$ (CWA; see Section 2).

Our main contributions towards our vision of an automated, provenance-driven security audit infrastructure for Open Source software are:

- We give an overview of static code analysis, which we use for our purpose (Section 3).

- We describe our method for querying the development process by using provenance (Section 4).

- We outline our ongoing efforts on combining information from process provenance with static code analysis for some specific revisions of the source code (Section 5).

\footnotetext{
${ }^{1}$ https://www.apple.com/covid19/contacttracing

${ }^{2}$ https://github.com/corona-warn-app
} 


\section{DEVELOPMENT OF THE “CORONA-WARN-APP”}

The development of the Corona-Warn-App gets special attention during the COVID-19 pandemic; the development had to be done in a short time frame: development started in April 2020 and the app was released on $16^{\text {th }}$ June, 2020 for Android and iOS. CWA is developed by SAP and Telekom using a transparent and open development process. CWA has a decentralized architecture, accompanied by centrally-managed Java-based server applications to distribute findings about infected users and store test results uploaded by the laboratories.

CWA development history is publicly available from 12 repositories (some of them auxiliary), including data since April 29th, for source code changes (5,624 git commits; Figure 1), issue tracking (1,397 GitHub issues) and code review (2,144 GitHub pull requests) ${ }^{3}$. The human team participating in the development is composed of 306 persons authoring code changes. Having into account the short time span, this amounts to a considerable effort, and suggests that most of the real activity is shown in these public repositories.

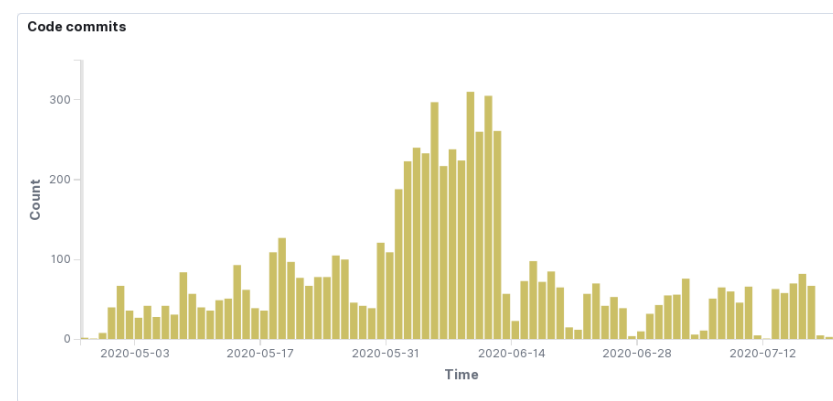

Figure 1: Code commits for the Corona-Warn-App repositories over time.

The analysis of the software development context for applications, by retrieving metadata from software repositories, has been an active area of research since the early 2000s [14, 21]. During this time several tools have been developed to get some metrics about the software development process and the team building it. We use GrimoireLab ${ }^{4}$, a toolset for retrieving data from software development repositories, store it, and perform some analytics via its SaaS instance Cauldron ${ }^{5}$ to produce statistics for the CWA. In this case, the context analysis ensures that the data analyzed for provenance is likely real (e.g., it is not likely that the analyzed repositories are not "dump repositories", where code is copied from time to time, while the real activity happens elsewhere), and gives an idea of the volume of activity caused by the project. In a more complete analysis, software development analytics may complement our provenance analysis by providing insights about how the different actors behave in the project, and how their contributions are related and processed.

\footnotetext{
${ }^{3}$ All numbers are for July 20 th.

${ }^{4}$ GrimoireLab: http://chaoss.github.io/grimoirelab

${ }^{5}$ Cauldron: https://cauldron.io
}

\section{CODE AUDIT WITH STATIC ANALYSIS}

Static code analysis is a proven method for program analysis and can be used as an early indicator for identifying pre-release defects [11].

Static analysis of program code spans a spectrum of tools, ranging from linters, which check adherence of code to coding standards on a syntactical level, to full-fledged verification tools, which formally prove properties of the code. Checked properties also cover multiple aspects of program code, including null pointer errors, memory-related errors, concurrency bugs, taint-related problems (i.e., data leaks and injection vulnerabilities). In our analysis, we static analysis tools for Java, Kotlin, and Android (Table 1).

Table 1: Used static analysis tools.

\begin{tabular}{ll}
\hline Static analysis tool & Category \\
\hline Xanitizer & taint analysis \\
infer & formal verification \\
Spotbugs & coding rules \\
Detekt & coding rules \\
Checkstyle & linter, coding rules \\
Flowdroid & taint analysis \\
SonarQube & linter, coding rules \\
\hline
\end{tabular}

Static analysis tools can be integrated at various points in a developer's lifecycle, while coding in terms of IDE plugins, when committing to a developer repository, either in batch mode or at diff-time, or when conducting quality insurance.

The usability of static analysis is known to be influenced by factors such as false-positive ratio, understandable and actionable analysis results, and integration with developer workflow [7, 16]. Experiences in large-scale application of static analysis shows, that integration with developer workflow and reporting bugs as soon as possible is important.

For example, SonarCloud found a bug, which was introduced to the repository "cwa-app-android" by the pull request \#876 (Figure 2$)^{6}$. The bug was found by SonarScanner before the pull request was accepted and the appropriate line should be deleted. The variable denomination fakeHeader gives a further hint, that this code lines are probably debug code and should not be part of production code.

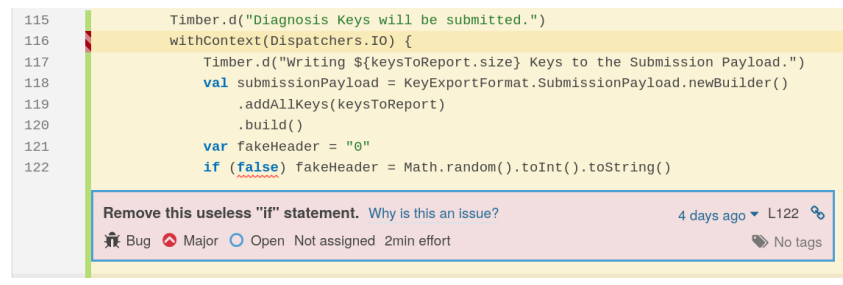

Figure 2: Introduced bug 'CWE-561 - Dead code', 'CWE-570 expression is always false', detected by SonarQube Scanner.

\footnotetext{
${ }^{6}$ Pull request \#876 was no longer available at the time of publication. Other issues found in the repository cwa-app-android by Sonarcloud are here: https://sonarcloud. io/project/issues?id=corona-warn-app_cwa-app-android
} 


\section{PROVENANCE OF REPOSITORIES}

Software development is a highly complex process involving a wide range of responsibilities and people. In addition the complexity of the software itself grows over time. To cope with this different tools are used to support the development process. During the entire software development process, all these support tools produce several types of data. These large amounts of data, which are generated before, during, and after the development of a software, can be analyzed using provenance [10].

Provenance analysis focusing on the development of open source software projects provides insight into the interactions of people. These interactions can fall into different categories. The most notable interactions in the development of track and trace software for COVID-19 are those that scrutinise the nature of the data collected and stored, which is hard for automated processes alone to evaluate ethical considerations. This can be evident in the provenance by the number of people collaborating outside of the development team, the number of developers, and the issues reported. While these types of measures cannot guarantee the ethics of the software, it does provide an indication that it has been evaluated by humans.

\subsection{Generating Retrospective Provenance for git Repositories}

To analyze the development process, we extract retrospective provenance [9] from repositories and store it in a graph database for further analysis (Figure 3) [15]. To extract provenance from git-based

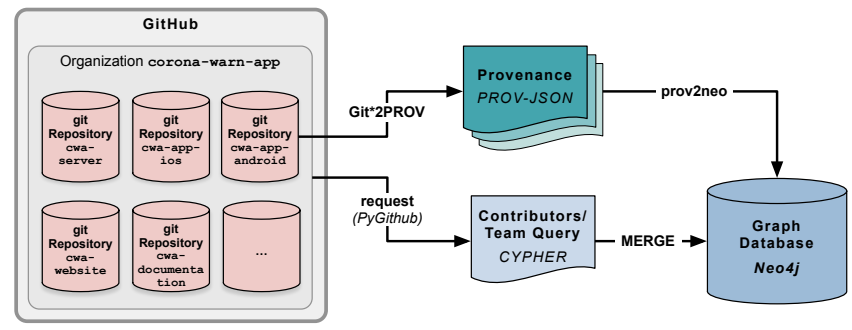

Figure 3: Extracting provenance from git repositories.

projects we use tools, which crawl the git repositories and additional information, such as issues or pull requests (Git2PROV [3, 19] and GitHub2PROV [13]). The provenance is generated as a file in JSON format and then stored in a Neo4j graph database. We note that while GitHub already provides visualisations for their hosted projects, the GitHub2PROV model supports bespoke visualisations that benefit from complex queries across the model's graph structure, which are not achievable using GitHub's API.

\subsection{Using and Analyzing Provenance}

To analyze the provenance graph, many visual and analytical methods exist; including semantic reasoning. For example, we illustrate querying and using the provenance graph for a simple example query for the CWA repository "cwa-server": "Which files have commits by team members as well as external contributors?"

We generate a CYPHER query, that adds information about contributors roles. We retrieve member information via the GitHub API and store it in Python lists of team members and external contributors, which we insert in a CYPHER template. This CyPHER query creates new directed relations between persons (PROV Agents) and files (PROV Entities); for example, the relation for team members is: (:Agent)-[: CONTRIBUTES_TO \{role: 'team'\}]->(:Entity).

Then we query for files, where team members and external contributor made changes at any of the files revisions (Listing 1). The query result is exported, either for visualization or as input for the static code analysis (Section 5).

Listing 1: Find all files where a team member AND an external contributor contributed changes.

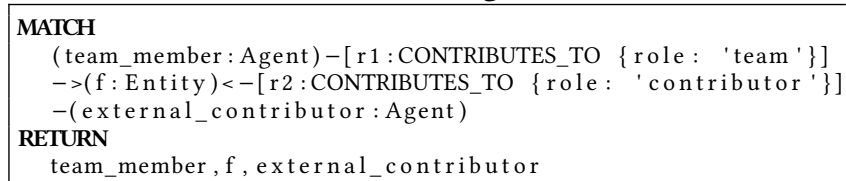

\section{RETROSPECTIVE CODE ANALYSIS FOR OPEN SOURCE SOFTWARE PROJECTS}

For conducting a security analysis of the CWA and its development process, we integrate the extracted provenance (Section 4) with bugs or vulnerabilities as reported by the selection of static analysis tools (Section 3). In our infrastructure (Figure 4), we therefore consider individual commit snapshots in the history of the CWA repositories. According to the respective repository, we run certain static analysis tools on a snapshot, track their reported findings and save them into a database for later analysis.

Due to the various involved static analysis tools and their differing report formatting and output granularity, the tools' findings need to be consolidated such that, for example, duplicated findings can be identified. The tools' reports are therefore parsed to extract the locations and types of found bugs or vulnerabilities; the latter is additionally normalized using the Common Weakness Enumeration $(\mathrm{CWE})^{7}$ and other bug ontologies. Interlinking the tools findings with provenance information is done via the respective snapshot's commit hash.

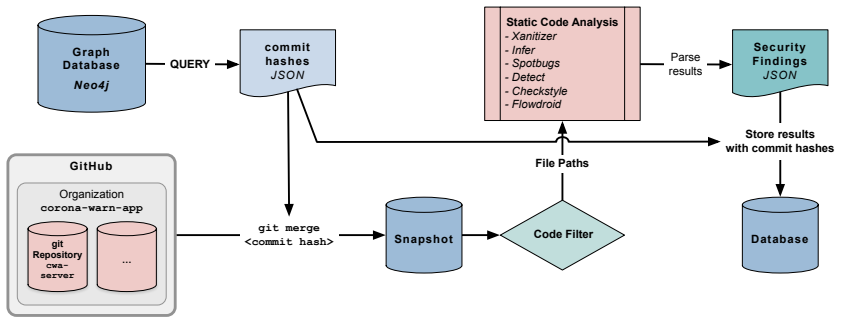

Figure 4: Commit-hash related security analysis.

Using the combined information then allows various questions for researching on the CWA development process and how security has been addressed. For instance:

- Classical hypotheses of empirical software engineering, like on the correlation of repository metrics as code churn and

\footnotetext{
${ }^{7}$ https://cwe.mitre.org/
} 
the number of found vulnerabilities or bugs [12], can be tested for the CWA case study.

- The usage of static analysis tools can be investigated, answering questions like how effective certain tools-or combinations thereof-were in uncovering bugs or vulnerabilities [5] or how understandable and usable their reports were [7].

- Characteristics of the vulnerability management in the CWA app development process can be analyzed quantitatively, using metrics like mean time to fix [6], or qualitatively, using fault tree analysis.

\section{RELATED WORK}

Baumgärtner et al. [1] categorized occurring security and privacy risks of existing contact tracing app solutions from a methodological point of view. They discussed different architectures, conducted an experimental study, and created a movement profile of an infected person with an early version of the DP3T app. A similar work by Vaudenay [18] describes the data exchange of the decentralized DP3T solution and possible attack scenarios on the communication, which is always possible without hyperlocal data. He concludes, that there are downsides in the design of decentralized apps and shows improvements. Both works did not focus on the software development itself.

Sun et al. [17] investigate the security of contact tracing applications by the use of static and dynamic analysis tools. They criticised, that not all contact tracing app developers make their code publicly available.

Wang et al. [20] analyzed the activities of much-contributing developers to open source projects in an empirical study and looked also on other repository artifacts besides the code. They investigated the communication between developers and quality of software with increasing contribution.

\section{CONCLUSIONS AND FUTURE WORK}

We described our vision for automated, provenance-driven security analysis of git-based software repositories. A provenance graph helps to discover hidden information from software repositories and pinpoint to code changes where static analysis tools should applied.

In the future, we apply our method on various software projects where security of the software product is essential. This includes developing tools and visualizations for developers to investigate how software is developed, the processes used, and the details around how security issues are identified and fixed.

Another future work is to capture code insertions and deletions of individual commits by diff trees [4]. This would enable us to enrich the provenance information; not just with the static code view, via the analysis of commit snapshots, but also with a dynamic view. As a result, sources and fixes of vulnerabilities identified by static analysis could be better researched.

\section{ACKNOWLEDGMENTS}

We thank DLR's student research assistant Claas de Boer (TU Dresden) for his tool prov2neo.

\section{REFERENCES}

[1] Lars Baumgärtner, Alexandra Dmitrienko, Bernd Freisleben, Alexander Gruler, Jonas Höchst, Joshua Kühlberg, Mira Mezini, Markus Miettinen, Anel Muhamedagic, Thien Duc Nguyen, Alvar Penning, Dermot Frederik Pustelnik, Filipp Roos, Ahmad-Reza Sadeghi, Michael Schwarz, and Christian Uhl. 2020. Mind the GAP: Security \& Privacy Risks of Contact Tracing Apps. (2020). arXiv:2006.05914v1 [cs.CR]

[2] Noel Carroll and Kieran Conboy. 2020. Normalising the "new normal": Changing tech-driven work practices under pandemic time pressure. International fournal of Information Management (2020).

[3] Tom De Nies, Sara Magliacane, Ruben Verborgh, Sam Coppens, Paul Groth, Erik Mannens, and Rik Van De Walle. 2013. Git2PROV: Exposing Version Control System Content as W3C PROV. In Proceedings of the 12th International Semantic Web Conference (Posters \& Demonstrations Track) - Volume 1035 (ISWC-PD '13). CEUR-WS.org, 125-128.

[4] Jean-Rémy Falleri, Floréal Morandat, Xavier Blanc, Matias Martinez, and Martin Monperrus. 2014. Fine-grained and accurate source code differencing. In ACM/IEEE International Conference on Automated Software Engineering, ASE '14, - September 15-19, 2014. ACM, Vasteras, Sweden, 313-324.

[5] Andrew Habib and Michael Pradel. 2018. How many of all bugs do we find? a study of static bug detectors. In Proceedings of the 33rd ACM/IEEE International Conference on Automated Software Engineering, ASE 2018, Montpellier, France, September 3-7, 2018. ACM, 317-328. https://doi.org/10.1145/3238147.3238213

[6] Rattikorn Hewett and Phongphun Kijsanayothin. 2009. On modeling software defect repair time. Empirical Software Engineering 14 (04 2009), 165-186.

[7] Rohan Krishnamurthy, Thomas S. Heinze, Carina Haupt, Andreas Schreiber, and Michael Meinel. 2019. Scientific developers v/s static analysis tools: vision and position paper. In Proceedings of the 12th International Workshop on Cooperative and Human Aspects of Software Engineering, CHASE@ICSE 2019, Montréal, QC, Canada, 27 May 2019. IEEE/ACM, 89-90.

[8] Rohan Krishnamurthy, Michael Meinel, Carina Haupt, Andreas Schreiber, and Patrick Mäder. 2018. DLR Secure Software Engineering: Position and Vision Paper. In Proceedings of the 1st International Workshop on Security Awareness from Design to Deployment (Gothenburg, Sweden) (SEAD '18). ACM, 49-50.

[9] Timothy McPhillips, Shawn Bowers, Khalid Belhajjame, and Bertram Ludäscher. 2015. Retrospective Provenance without a Runtime Provenance Recorder. In Proceedings of the 7th USENIX Conference on Theory and Practice of Provenance (Edinburgh, Scotland) (TaPP'15). USENIX Association, USA, 1.

[10] Luc Moreau, Paul Groth, Simon Miles, Javier Vazquez-Salceda, John Ibbotson, Sheng Jiang, Steve Munroe, Omer Rana, Andreas Schreiber, Victor Tan, and Laszlo Varga. 2008. The provenance of electronic data. Commun. ACM 51, 4 (2008), 52-58.

[11] Nachi Nagappan and Thomas Ball. 2005. Static analysis tools as early indicators of pre-release defect density. In Proceedings. 27th International Conference on Software Engineering, 2005. ICSE 2005. ACM, 580-586.

[12] Nachiappan Nagappan and Thomas Ball. 2005. Use of relative code churn measures to predict system defect density. In 27th International Conference on Software Engineering (ICSE 2005), 15-21 May 2005. ACM, St. Louis, Missouri, USA, 284-292.

[13] Heather S. Packer, Adriane Chapman, and Leslie Carr. 2019. GitHub2PROV: Provenance for Supporting Software Project Management. In Proceedings of the 11th USENIX Conference on Theory and Practice of Provenance (Philadelphia, PA, USA) (TAPP'19). USENIX Association, USA, 1.

[14] Gregorio Robles, Jesus M. Gonzalez-Barahona, and Juan Julian Merelo. 2006. Beyond source code: The importance of other artifacts in software development (a case study). Journal of Systems and Software 79, 9 (2006), 1233-1248. Fourth Source Code Analysis and Manipulation Workshop (SCAM 2004).

[15] Andreas Schreiber and Claas de Boer. 2020. Modelling Knowledge about Software Processes using Provenance Graphs and its Application to Git-based Version Control Systems. In 42nd International Conference on Software Engineering Workshops. IEEE/ACM, Seoul, Republic of Korea.

[16] Justin Smith, Lisa Nguyen Quang Do, and Emerson Rex Murphy-Hill. 2020. Why Can't Johnny Fix Vulnerabilities: A Usability Evaluation of Static Analysis Tools for Security. In Sixteenth Symposium on Usable Privacy and Security (SOUPS)

[17] Ruoxi Sun, Wei Wang, Minhui Xue, Gareth Tyson, Seyit Camtepe, and Damith Ranasinghe. 2020. Vetting Security and Privacy of Global COVID-19 Contact Tracing Applications. arXiv:2006.10933 [cs.CR]

[18] Serge Vaudenay. 2020. Analysis of DP3T. Cryptology ePrint Archive, Report 2020/399. https://eprint.iacr.org/2020/399.

[19] Ruben Verborgh, Sara Magliacane, Andreas Schreiber, and Vlad Korolev. 2020. onyame/Git2PROV: Improved error handling. https://doi.org/10.5281/zenodo. 3942169

[20] Zhendong Wang, Yang Feng, Yi Wang, James A. Jones, and David Redmiles. 2020. Unveiling Elite Developers' Activities in Open Source Projects. ACM Trans. Softw. Eng. Methodol. 29, 3, Article 16 (June 2020). https://doi.org/10.1145/3387111

[21] Thomas Zimmermann, Peter Weisgerber, Stephan Diehl, and Andreas Zeller. 2004. Mining Version Histories to Guide Software Changes. In Proceedings of the 26th International Conference on Software Engineering (ICSE '04). IEEE, 563--572. 